\section{Sexualidad infantil: información para orientar la práctica clínica}

\section{RESUMEN}

La sexualidad sigue considerándose un tema tabú en nuestra sociedad; como tal, tiende a ocultarse o a escindirse del cuerpo. Proporcionar información sobre sexualidad es parte indispensable de una educación integral, necesaria para el desarrollo saludable las personas. La sexualidad está presente durante todo el ciclo vital del ser humano y se encuentra en permanente proceso de transformación. No tiene una sola función, se trata de un proceso dinámico en constante evolución. En menores de edad tiene una serie de características que la hacen diferente a los adultos, por ello, el objetivo de este trabajo es proporcionar un marco de referencia sobre el desarrollo psicosexual infantil con la finalidad de invitar a la búsqueda de mayor información y capacitación sustentada científicamente.

Palabras clave: sexualidad infantil, desarrollo integral, etapas de la sexualidad, tabú.

\section{Child sexuality: information to guide the clinical practice}

\begin{abstract}
Sexuality is still considered taboo in our society; as such, it tends to hide or excised from the body. To provide information on sexuality is an essential part of a comprehensive education, necessary for healthy people development. Sexuality is present throughout the life cycle of human beings and is in the process of transformation. It does not have a single function; It is a dynamic process in constant evolution. In children it has a number of features that make it different from to adults, therefore, the aim of this paper is to provide a frame work on child psychosexual development in order to promote seeking more information and scientifically supported training.
\end{abstract}

Key words: Infantile sexuality, integral development, stages of sexuality, taboo.
Corina A. García-Piña ${ }^{1}$

${ }^{1}$ Pediatra, coordinadora de la Clínica de Atención Integral al Niño Maltratado, Instituto Nacional de Pediatría, México.

Recibido: 2 de marzo del 2015

Aceptado: 25 de agosto del 2015

Correspondencia: Dra. Corina Araceli García-Piña Clínica de Atención Integral al Niño Maltratado Instituto Nacional de Pediatría Insurgentes Sur 3700-C CP 04530, México, D.F. Tel: 01840900 ext. 1413 corinagarcia_9@yahoo.com.mx

Este artículo debe citarse como García-Piña CA. Sexualidad infantil: información para orientar la práctica clínica. Acta Pediatr Mex 2016;37:47-53. 


\section{INTRODUCCIÓN}

La Organización Mundial de la Salud reporta que la salud sexual es un estado de bienestar físico, emocional, mental y social relacionado con la sexualidad; no es sólo la ausencia de enfermedad, de disfunción o de malestar; para lograrlo se requiere un acercamiento positivo y respetuoso con las y los niños hacia el tema, así como la posibilidad de que adquiera un desarrollo saludable, libre de coerción, discriminación y violencia. Para que esto sea posible los derechos humanos y sexuales de todas las personas deben ser respetados. La sexualidad se encuentra en todas las fases de la vida y se desarrolla a través de un proceso dinámico, gradual y continuo. ${ }^{1}$

Proporcionar información sobre sexualidad es parte indispensable de una educación integral, necesaria para el desarrollo armónico de las personas. Una educación sexual apropiada debe respetar las diferentes etapas del desarrollo y promover valores tales como la equidad, la privacidad, el respeto y la responsabilidad.

El tema de sexualidad sigue siendo un tabú en nuestros días; es frecuente que en la familia y otros entornos en donde interactúan las y los niños se muestren actitudes de desaprobación hacia ciertas manifestaciones propias del desarrollo. En este sentido, las actitudes evasivas o el silencio también son una forma de transmitir información sobre sexualidad mediante mensajes de censura o prohibición. ${ }^{2,3}$

Por otro lado, los medios de comunicación tienen un impacto importante en su desarrollo sexual de los menores de edad, ya que hoy en día se encuentran expuestos (cada vez a menor edad) a manifestaciones erróneas, distorsionadas e incomprensibles de la sexualidad. Existe una gran facilidad para leer contenidos inapropiados y, además, la supervisión de las actividades en internet por parte de los progenitores suele ser insuficiente. Por ello no es posible mantener un lenguaje oculto o actitudes evasivas hacia el tema; es necesario proporcionar información para que vayan construyendo una sexualidad saludable y estén preparados para los cambios que su cuerpo y emociones van experimentando; que comprendan el significado de la privacidad e intimidad, que aprendan sobre el respeto hacia su cuerpo y el de los demás, que se sientan libres de hacer preguntas y que puedan expresar conductas saludables acordes con su edad. No será con silencio, prejuicios o temores como podremos ayudarlos. ${ }^{4}$

La educación sexual integral es necesaria para un desarrollo saludable; es un derecho de todos los seres humanos independientemente de su edad, sexo, raza o religión; ninguna creencia, ideología o dogma debe estar por encima de una educación sexual saludable orientada científicamente. Todas las personas que participamos, de una u otra manera, en la formación de niñas y niños, tenemos la responsabilidad de conocer y ofrecer una educación sexual integral adaptada a la edad y que permita un desarrollo saludable.

\section{OBJETIVO}

Es frecuente que madres y padres de familia acudan con profesionales del área médica para solicitar información ante las conductas relacionadas con la sexualidad de sus hijas e hijos. El objetivo de este trabajo es proporcionar un marco de referencia sobre desarrollo psicosexual infantil y será responsabilidad del profesional buscar mayor capacitación.

\section{CONCEPTOS SOBRE SEXUALIDAD HUMANA}

Sexualidad: conjunto de manifestaciones fisiológicas, anatómicas, psicológicas, afectivas y sociales del ser humano; incluye al sexo, al género, al erotismo, a la vinculación afectiva y a la reproducción. ${ }^{1,5}$ 
Sexo: se refiere a las características biológicas que definen a los seres humanos como hombre o mujer. ${ }^{1,5}$

Género: conceptos o construcciones sociales de las funciones, roles, comportamientos, actividades y atributos que se asignan a las personas por ser hombres o mujeres. ${ }^{1,5}$

Zona erógena: región del cuerpo que funcionalmente es sitio de placer y gratificación sexual: zona oral, genital, anal. ${ }^{6}$

Sublimación: proceso psíquico mediante el cual la energía sexual se canaliza hacia otras actividades como la creación artística, el trabajo intelectual, la creatividad y la vida social. ${ }^{6}$

\section{ETAPAS DE LA SEXUALIDAD}

La sexualidad está presente durante todo el ciclo vital y se encuentra en permanente proceso de transformación, no tiene una sola función ni se presenta como un periodo en la vida del ser humano. Es un proceso dinámico en donde la energía sexual se organiza por distintas etapas y cada una tiene sus peculiaridades; estas etapas son indispensables para ir formando la personalidad adulta y alcanzar una sexualidad madura. ${ }^{7}$

A continuación se describen algunas manifestaciones y la edad aproximada de cada etapa; ${ }^{6} \sin$ embargo, es importante mencionar que no es el calendario el que marca los ritmos del desarrollo sino los procesos singulares que cada persona realiza a su tiempo y a su modo.

Fase oral: se presenta durante el primer año de vida. En esta etapa la boca es el núcleo primario de energía y gratificación sexual. Desde el nacimiento, sentimos placer al ser amamantados; la boca es la parte del cuerpo que proporciona satisfacción, agrado y acercamiento hacia la madre. En esta etapa, debido a que es el centro del placer, es común observar que los menores se lleven frecuentemente objetos a la boca, así como las manos y los pies. Además se inicia la etapa de conocer su propio cuerpo.

Fase anal: abarca del primer al tercer año de edad. La zona erógena se encuentra focalizada en la región anal. En esta etapa el niño adquiere mayor control de su cuerpo: inicia el control de esfínteres, cuyos músculos le permiten retener y expulsar las heces, y adquiere placer al hacerlo; además descubre que puede tener control del esfínter permitiendo el inicio al proceso de su independencia.

Fase fálica: de los tres a los cinco años de edad. El núcleo de energía sexual se encuentra en los genitales. Niñas y niños experimentan placer al tocarse los genitales y masturbarse. Se presenta el complejo de Edipo donde la libido o energía sexual está enfocada en los padres y son objetos de deseo.

Etapa de latencia: se presenta aproximadamente entre los seis y once años de edad. Este periodo comprende la declinación de las manifestaciones de la sexualidad infantil y el comienzo de la pubertad; se observa una disminución de las actividades relacionadas con el erotismo y aparecen los sentimientos de pudor, asco, las aspiraciones morales y estéticas. En esta etapa las manifestaciones de la sexualidad sufren una profunda transformación, sus intereses se dirigen hacia otros destinos, hacia el mundo extrafamiliar. En la latencia se da gran importancia a la integración con los pares pues pertenecer a un grupo es uno de los asuntos más importantes. En este período la sexualidad está presente de otra forma, la sublimación cede lugar a fortalecer otros aspectos como el aprendizaje de diferentes habilidades, las responsabilidades, la reflexión, el deseo por aprender, la recreación, el deporte y el fortalecimiento de los vínculos afectivos con su grupo de amigos. 
Etapa genital: etapa de la adolescencia en la que hay una serie de cambios físicos y psicológicos, continúa la lucha por la autonomía e identificación con el grupo de compañeros. Durante esta fase se alcanza la capacidad de reproducción; sin embargo, la madurez emocional y física no logran su totalidad.

\section{MANIFESTACIONES DE LA SEXUALIDAD INFANTIL}

Con frecuencia, la sexualidad se asocia exclusivamente con la genitalidad o el erotismo; no obstante, sus manifestaciones tienen una dimensión más amplia, la sexualidad está conformada integralmente e incluye también otros aspectos..$^{8-11}$

Manifestaciones relacionadas con el género: Relacionadas con pertenecer a uno u otro género, desde las diferencias físicas, la forma de expresarse y relacionarse con los demás. Se ha demostrado que la forma en que nos relacionamos con las niñas y los niños es diferente. La perspectiva en relación con el género en la mayoría de los progenitores se va construyendo desde el embarazo; cuando se descubre el sexo del bebé. En general, la manera de comunicarse y relacionarse con cada género es distinta, desde las expectativas que se van construyendo, la forma de hablarles, de expresarse o de jugar. ${ }^{11}$ Los infantes empiezan a comprender las diferencias entre varones y mujeres durante los primeros tres años de vida: el niño observa al modelo masculino que tiene más próximo y la niña al femenino. Van identificando que pertenecen a un determinado género e intentan imitar a los mayores, asumen los papeles observados en la familia, en la escuela, en sus ambientes cercanos y los reproducen (Cuadro 1); del mismo modo interiorizan los valores que estos roles adquieren en la sociedad; por ello es importante mostrar modelos respetuosos y equitativos de relación entre hombres y mujeres. Algunas de sus manifestaciones son imitar el rol de la mamá o del papá, investigar las diferencias entre hombres y mujeres a través de los juegos, en la convivencia familiar o con amigos. En este sentido es importante transmitir el concepto de equidad, enseñar y mostrar en la convivencia familiar y escolar que niños y niñas son valiosos y que tienen los mismos derechos, responsabilidades y oportunidades para desarrollarse, crecer y aprender.

Manifestaciones relacionadas con la reproducción: es la forma en que niñas y niños se identifican o están interesados en los eventos de la concepción, el embarazo y el parto. Se manifiesta por la vivencia de ser como mamá o como papá y se expresa en los juegos o en experiencias con familiares respecto a la maternidad o paternidad, al investigar y conocer del embarazo, sobre cómo se forman los bebés, cómo nacen, etcétera. Desde pequeños van experimentando y construyendo su capacidad de maternidad o paternidad con sus progenitores, en los juegos con sus iguales, en el cuidado de sus mascotas o juguetes; en este sentido, es necesario reconocer y permitir que los varones, al igual que las niñas, desarrollen y expresen plenamente esta capacidad, que no haya distinción entre juegos de niñas y niños, que se les permita experimentar y desarrollar su capacidad de maternidad y paternidad a través del juego. ${ }^{12}$

Manifestaciones sobre los vínculos afectivos: es la capacidad de relacionarse y sentir afecto por seres queridos, familiares, amigos y mascotas. Los vínculos afectivos se relacionan con la capacidad de sentir afecto por los demás. El primer vínculo es con la madre, el afecto y los estímulos transmitidos al bebé serán la pauta para ir desarrollando su capacidad de vinculación. En los bebés las experiencias sensoriales son básicas para el desarrollo. Los adultos deberán enseñar y mostrar desde las primeras etapas formas de vinculación salu- 
García-Piña CA. Sexualidad infantil

Cuadro 1. Manifestaciones saludables de la sexualidad infantil

\begin{tabular}{|c|c|}
\hline $\begin{array}{l}\text { Relacionadas con } \\
\text { el género }\end{array}$ & $\begin{array}{l}\text { - Curiosidad y preguntas frecuentes sobre la diferencia entre niños y niñas } \\
\text { - Exploración de genitales: buscar, tocar sus genitales o de otros niños y niñas, observan y les } \\
\text { Ilaman la atención sus diferencias } \\
\text {. } \quad \text { Juegan al papá y a la mamá } \\
\text { - Niñas y niños se visten con ropas de los progenitores, independientemente del sexo } \\
\text { - } \quad \text { Juegan y pueden vestirse o expresarse como personas del sexo opuesto } \\
\text { Curiosidad por observar o tocar el cuerpo de los adultos }\end{array}$ \\
\hline $\begin{array}{l}\text { Vínculos } \\
\text { afectivos }\end{array}$ & $\begin{array}{l}\text { - Interés por tener novio o novia (con amigos, familiares o personajes favoritos) } \\
\text { - Curiosidad por el matrimonio (las personas cercanas o queridas son elegidas como "novio" } \\
\text { o "esposo") } \\
\text { - Interés por crecer, enamorarse y tener una pareja } \\
\text { - } \quad \text { Sufren por rechazos afectivos entre sus iguales } \\
\text {. } \quad \text { Cuidan, alimentan y brindan afecto a sus juguetes y mascotas } \\
\text {. } \\
\text { Temorés o preocupación por el divorcio o separación de los progenitores }\end{array}$ \\
\hline Erotismo & $\begin{array}{l}\text { Estimular sus genitales con la mano o frotándolos con objetos, muebles o juguetes } \\
\text { Juegos sexuales: niños y niñas de edad similar juegan a acercar sus cuerpos para verlos y } \\
\text { tocarlos } \\
\text {. Se abrazan y besan entre edades similares } \\
\text { Juegan al doctor, los esposos, exploran su cuerpo y los genitales }\end{array}$ \\
\hline Reproducción & 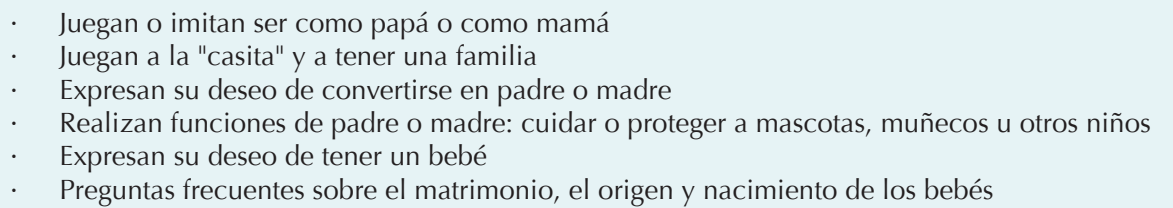 \\
\hline
\end{tabular}

dable. Cuando los progenitores o cuidadores expresan cariño al tocar, acariciar, alimentar o hablar al bebé sientan las bases para brindarle seguridad, aspecto fundamental en su desarrollo; la familia es la principal portadora de vínculos afectivos. Conforme avanza el desarrollo y la socialización se van formando vínculos con amigos a través de los juegos; empiezan solitarios, posteriormente juegan en compañía pero dispersos y, alrededor de los 6 años, el juego ya es en equipo, entonces los menores empiezan a comprender y a respetar los límites; los amigos comienzan a formar parte esencial de su vida.

Es frecuente que mencionen querer casarse con sus progenitores, con algún amigo o familiar, esto puede generar confusión en los adultos al interpretar eróticamente estas expresiones, que son parte de su capacidad de amar y formar vínculos afectivos. En este contexto, es importante tener claro, informar y mostrar el rol que ocupan los hijos y los padres en las relaciones familiares. ${ }^{13}$ Mostrar relaciones saludables y respetuosas, que niñas y niños aprendan a expresar sus sentimientos y a construir relaciones basadas en el respeto a sí mismos y hacia los demás, mostrando modelos afectivos dentro de la familia.

Manifestaciones relacionadas con el erotismo: reflejan la capacidad que tienen niños y niñas de percibir las diferentes sensaciones corporales. El recién nacido tiene una gran estimulación afectiva a partir del primer vínculo con la madre, percibe las sensaciones y mediante el contacto va desarrollando su erotismo a través del reflejo de succión, las miradas, las caricias y la proximidad con su cuerpo. Durante los primeros meses va descubriendo su cuerpo y las sensaciones que puede experimentar con los diferentes sentidos: la vista, el olfato, el oído, el tacto y el gusto; los sentidos también le ayudan 
a conocerse y a relacionarse con personas y objetos e ir diferenciando las emociones que le provocan placer o disgusto.

La masturbación es parte del desarrollo; se inicia a partir de que descubre la zona genital y posee mayor coordinación motora entre los tres y los cinco años de edad; disminuye en la etapa de latencia. Se recomienda transmitir el concepto de privacidad, explicar que la masturbación es parte de la propia intimidad, que es un acto privado y que puede hacerse en un lugar seguro, con las manos limpias y nunca frente a otras personas; informarles que nadie debe tocar su cuerpo y que también deberán respetar el cuerpo de los demás. Se recomienda no mostrar enojo, conductas de desaprobación o castigos. A través de la masturbación los infantes van descubriendo, explorando y experimentando sensaciones en su cuerpo. ${ }^{10}$

Es necesario que desde pequeños aprendan a nombrar su genitales con el nombre correcto (vulva, pene, testículos); un buen momento para ello suele ser durante el cambio de pañal o al bañarlos. Es común que se asignen nombres incorrectos a los genitales o que hablar de ellos provoque incomodidad, lo cual puede transmitir el mensaje de que existe algo "malo" o "extraño" en ellos y no tener confianza para cuidar o hablar de "esa zona" del cuerpo que causa incomodidad o genera conflicto.

Entre los tres y cinco años de edad suelen mostrar gran curiosidad por conocer las partes de su cuerpo. En esta edad es común que exploren y toquen su cuerpo y sus genitales, se dan cuenta de cómo son, descubren las similitudes y diferencias entre niñas y niños. Jugar con los genitales es parte del proceso, jugar al doctor, a la mamá y al papá o hacer comparaciones entre niñas y niños, masturbarse o frotarse los genitales con juguetes $u$ otros objetos es parte del desarrollo de su sexualidad.

\section{CONCLUSIONES}

La sexualidad sigue considerándose un tema tabú y como tal se tiende a ocultarlo o a escindirlo del cuerpo; la sexualidad de niños y niñas tiene una serie de características que la hacen diferente a la de los adultos; más aún, las expresiones de la sexualidad reflejan el propio desarrollo, los aspectos afectivos, emotivos, de conocimiento y socialización tienen una gran relevancia para su crecimiento. Los juegos sexuales de este periodo se basan en la curiosidad, el conocimiento y la experiencia de sensaciones corporales. El establecimiento de vínculos afectivos satisfactorios y sin violencia proporcionan un referente para un desarrollo armónico en la edad adulta. ${ }^{13}$

La sexualidad infantil se expresa de diversas maneras, las niñas y niños saludables juegan y conocen su cuerpo, tienen inquietudes sobre el amor, los besos, la diferencia entre niños y niñas, sobre cómo se hacen y nacen los bebés, etcétera. Estas son expresiones que requieren una participación activa de los progenitores para su orientación y para permitir que la comunicación, la confianza, la verdad y el respeto sean los ejes de la educación sexual en la familia. Todo tipo de educación sexual tendrá que basarse en el conocimiento científico y estar encaminado a proporcionar un desarrollo integral saludable, además de fomentar roles de género que promuevan el respeto y las relaciones justas y equitativas entre niñas y niños.

Agradecimiento:

Un especial agradecimiento a la psicóloga Sandra Posadas Pedraza por sus observaciones y comentarios en el trabajo.

\section{REFERENCIAS}

1. Organización Mundial de la Salud (OMS). (2006). Defining sexual health Report of a technical consultation on sexual health 28-31 January 2002. Ginebra. 
García-Piña CA. Sexualidad infantil

2. Barr ME, Zichele Moore J, Tammie Johnson J, Forrest JP. New Evidence: Data Documenting Parental Support for Earlier Sexuality Education. Journal of School Health 2014;84:10-17.

3. Thornton AC, Collins JD. Teaching parent to talk to their children about sexual topics. Clinics in Family practice. 2004;6:801-819.

4. Cabello Cádiz P, Fernández Villanueva I. La tecnología en la preadolescencia y adolescencia. Usos riesgos y propuestas desde las y los protagonistas. Save The Children. 2010. http://www.savethechildren.es/det_notyprensa. php?id=279\&seccion $=$ Not

5. World Association for Sexual Health; Organización Panamericana de la Salud (OPS) (2000). Promoción de la Salud Sexual Recomendaciones para la acción. Antigua: OPS.

6. Freud, S. (1905) Tres ensayos de teoría sexual. En: Obras Completas, Buenos Aires, Amorrortu Editores, 1998. V. 7.
7. Haberland NM, Roodow D. Sexuality Education: Emerging Trends in Evidence and Practice. Journal of adolescent health 2015;56:S15-S21.

8. Rubio, A. E. (1998). Introducción al estudio de la sexualidad. En Pérez, F. C. J.; Rubio, A. E. (Eds.) Antología de la sexualidad humana. 2a ed. (pp. 17-46). México: Conapo. (trabajo original publicado en 1994).

9. DeLamater J, Friedrich WN. Human sexual development. J Sex Res 2002;39:10-4.

10. American Academy of Pediatrics. Sexuality education for children and adolescents. Pediatrics 2001;108:498-502.

11. Elia PJ, Tokunaga J. Sexuality education: implications for health, equity, and social justice in the United States. Health Education 2015;115:105-120.

12. Duncan P, Dixon RR, Carlson J. Childhood and adolescent sexuality. Pediatr Clin N Am 2003;50:765-80.

13. Snegroff $\mathrm{S}$. No sexuality education is sexuality education. Fam Plann Perspect 2000;32:257-8. 